Bio - grafía. Escritos sobre la Biología y su Enseñanza. ISSN 2027-1034

Edición Extraordinaria. p.p. 657 - 664

Memorias del IX Encuentro Nacional de Experiencias en Enseñanza de la Biología y la Educación Ambiental. IV Congreso Nacional de Investigación en Enseñanza de la Biología.

\title{
PRÁCTICAS DE ENSEÑANZA ALREDEDOR DE LAS COLECCIONES BIOLÓGICAS: HACIA LA CONFIGURACIÓN DEL MUSEO PEDAGÓGICO DE BIOLOGÍA ${ }^{1}$
}

\author{
Francisco Medellín Cadena ${ }^{2}$ \\ Deysi Serrato Rodríguez ${ }^{3}$
}

\section{RESUMEN}

El presente tejido escritural emerge del proyecto de investigación "Estrategia pedagógica para la enseñanza de la vida y lo vivo a partir de las colecciones biológicas: Hacia la configuración del Museo Pedagógico de Biología" cuyo objetivo es visibilizar algunas prácticas de enseñanza alrededor de las colecciones en el Departamento de Biología de la Universidad Pedagógica Nacional, con miras a proponer elementos para la configuración del museo pedagógico de biología. Todo esto se consolidará a partir de la sistematización de prácticas pedagógicas alrededor de las colecciones, las que se constituyen en una apuesta pedagógica en el marco del Proyecto Curricular Licenciatura en Biología, a propósito de la formación de maestros.

Para la realización de esta investigación se toman elementos de la mirada arqueológica - genealógica que entran en diálogo con una perspectiva cualitativa, así se recurre al levantamiento del archivo y tematización del mismo desde las relaciones saber, poder y subjetivación, identificando elementos de orden histórico, pedagógico y didáctico que contribuyan a la propuesta de Museo Pedagógico de Biología.

Dentro de los primeros hallazgos, se identifican tres tendencias: la producción de materiales educativos, ejercicios de sistemática y taxonomía y propuestas pedagógicas alrededor de museo y colecciones. De igual forma, se encuentra en

\footnotetext{
${ }^{1}$ Las reflexiones que se presentan en esta ponencia emergen de los tránsitos investigativos en el marco del proyecto CIUP "Estrategia pedagógica para la enseñanza de la vida y lo vivo a partir de las colecciones biológicas: Hacia la configuración del Museo Pedagógico de Biología." Código DBI- 460-17

${ }^{2}$ Licenciado en Biología. Profesor Departam ento de Biología. Coordinador Grupo de Investigación CASCAD A. Departamento de Biología. Universidad Pedagógica Nacional.

${ }^{3}$ Licenciada en Biología. Especialista en Pedagogía. Magister en Educación. Profesora Departamento de Biología. Coordinadora Línea de Investigación Trayectos y Aconteceres. Universidad Pedagógica Nacional.
} 
Bio - grafía. Escritos sobre la Biología y su Enseñanza. ISSN 2027-1034

Edición Extraordinaria. p.p. 657 - 664

Memorias del IX Encuentro Nacional de Experiencias en Enseñanza de la Biología y la

Educación Ambiental. IV Congreso Nacional de Investigación en Enseñanza de la Biología.

construcción la comprensión de museo pedagógico como escenario dinámico, que se cruza con las nociones de memoria e historia en términos de la experiencia de los sujetos.

Finalmente, a modo de proyección se teje la propuesta de articular el museo pedagógico de biología con el Centro de Memoria de la UPN y el despliegue de alianzas y redes que potencien los procesos formativos y de proyección social de la Universidad Pedagógica Nacional.

Palabras clave: Museo, educación, enseñanza de la biología, colección

\section{ABSTRAC}

The following paper emerges from the research project "Pedagogical strategy for the teaching of life and living it from biological collections: Towards the configuration of the Pedagogical Museum of Biology" whose objective is visualize some teaching practices around the collections in the Department of Biology of the "Unversidad Pedagógica Nacional", with a view to proposing elements for the configuration of the pedagogical museum of biology. All this will be consolidated from the systematization of practices around the collections, which constitutes a pedagogical proposal within the framework of the "Proyecto Curricular Licenciatura en Biología" , on the subject of teacher training.

In order to carry out this research, we take elements of the archeological genealogical perspective that enter into dialogue with a qualitative perspective. This way, we use the archiving and thematization of the archive from knowledge, power and subjectivation, identifying elements of historical order, Pedagogical and didactic that contribute to the proposal of Pedagogical Museum of Biology.

Among the first findings, three trends are identified: the production of educational materials, systematics and taxonomic exercises, and pedagogical proposals around museum and collections. In the same way, is under construction the understanding of pedagogic museum as a dynamic scenario, it which intersects with the notions of memory and history in terms of the expe rience of the subjects.

Finally, as a projection is the proposal of articulating the pedagogical museum of biology with the Memory Center of the UPN and the deployment of alliances and networks that enhance the formative processes and social projection of the "Universidad Pedagógica Nacional"

Keywords: museum, education, teaching biology, collection 
Bio - grafía. Escritos sobre la Biología y su Enseñanza. ISSN 2027-1034

Edición Extraordinaria. p.p. 657 - 664

Memorias del IX Encuentro Nacional de Experiencias en Enseñanza de la Biología y la

Educación Ambiental. IV Congreso Nacional de Investigación en Enseñanza de la Biología.

\section{INTRODUCCIÓN}

El proyecto Curricular Licenciatura en Biología (PCLB) presenta como parte de su misión "La excelencia en la formación y cualificación de docentes para los diferentes niveles y modalidades del sistema educativo dentro de un contexto cultural, ético, pedagógico y científico, que responda a la formación integral del ciudadano que Colombia necesita." (PCLB, 2000, p: 17), lo cual permite pensar los procesos formativos en diversos escenarios siendo uno de ellos el Museo de Historia Natural y las colecciones biológicas, teniendo incidencia y brindando apoyo en los ciclos constitutivos del programa, ciclo de fundamentación y ciclo de profundización.

Así las cosas, el Museo de Biología, también llamado desde las prácticas "Casita de Biología", se constituye en un espacio donde se visibiliza la creación y transformación de prácticas alrededor de la enseñanza de la Biología, pues su relevancia histórica no sólo obedece a su tradición en la universidad o a la riqueza y abundancia de sus colecciones biológicas, sino preponderantemente como lugar de experiencias sobre los saberes acerca de la vida y lo vivo.

De este modo, aquellas experiencias se concretan en los informes de trabajo de grado, prácticas pedagógicas, proyectos de semestre, informes de investigación ante facultad y el Centro de Investigaciones (CIUP), espacios académicos, entre otros aspectos cuya reflexión central son las colecciones o el museo de biología como tal. Estos dejan entrever que la riqueza del museo está relacionada con la creación de experiencias sobre la enseñanza de la Biología que problematizan nociones sobre la vida, lo vivo, la biodiversidad, la diversidad biológica, el territorio y el patrimonio cultural.

Así se podría enunciar que la noción de colección ha sido enriquecida, no reduciéndose al almacenamiento de individuos preservados, sino que puede responder a otros tipos de materiales, que propenden por el respeto de la vida, elaborando prototipos, realizando colecciones de cantos, manuales ilustrados, biogeografía cultural, entre otros; así esto supera los saberes disciplinares científicos y visibilizan apropiaciones sociales de la biología que superan las tradiciones científicas y recogen los saberes locales y contextuales, de esta forma, se evidencia que la enseñanza de la biología, no es un remedo de la biología en tanto ciencia, no se reduce a lo útil y pertinente del conocimiento de lo vivo, por el contrario posibilita el encuentro de saberes que ponen en tensión lo vivo y la vida, y que sitúa la pregunta por cómo estos saberes son apropiados por los sujetos y cómo se materializan en sus mismas prácticas de vida.

De acuerdo con lo anterior, la propuesta de configurar el Museo Pedagógico de 
Bio - grafía. Escritos sobre la Biología y su Enseñanza. ISSN 2027-1034

Edición Extraordinaria. p.p. 657 - 664

Memorias del IX Encuentro Nacional de Experiencias en Enseñanza de la Biología y la

Educación Ambiental. IV Congreso Nacional de Investigación en Enseñanza de la Biología.

Biología abre la posibilidad de recrear y resignificar desde lo construido, en términos de reconocer la trayectoria del Museo de Biología en el marco de la Universidad, pero también, de dinamizar las prácticas tradicionales de la enseñanza de la biología con miras a problematizar su lugar desde lo contemporáneo. De esta manera, el museo entendido como escenario de reflexión pedagógica permite problematizar el saber biológico y su enseñanza, pues no obedece a una comprensión de lo vivo como capital para administrar o explotar; por el contrario, posiciona lo vivo en relación con la vida a propósito de una apuesta estética, ética y política, que aporta a la formación de maestros de manera particular, pero que acerca al público en general a realizar otros acercamientos con eso vivo que impregna su vida.

Por lo tanto, la apuesta de Museo Pedagógico de Biología supera la noción de museo como espacio que solo brinda información y conocimiento a propósito de la biodiversidad ecosistémica y organísmica, posicionándose como escenario que puede generar experiencias que pasen por los sujetos transformándolos y transformando sus dinámicas de vida. Así las cosas, estos elementos sitúan el museo como condición para problematizar la formación de maestros de biología y el lugar de las colecciones y otros escenarios en la enseñanza de la biología.

En ese sentido, para este caso en particular se presentan los primeros hallazgos en términos de la revisión de trabajos de grado y proyectos de facultad relacionados con colecciones y el museo de biología, y algunas reflexiones que se han ido construyendo desde las líneas de investigación Trayectos y Aconteceres y CASCADA a propósito de presentar elementos para pensar a modo de proyección el museo pedagógico de biología.

\section{METODOLOGÍA}

Para consolidar la propuesta investigativa se recurre a una perspectiva cualitativa que entra en diálogo con algunos elementos propuestos desde la caja de herramienta de la mirada arqueológica - genealógica. En esa vía, un posicionamiento desde lo cualitativo permite una investigación más dinámica, de caracteres holísticos y construida a partir de los contextos en los cuales se presentan los fenómenos, comprende las relaciones que tejen los sujetos, sus percepciones, intenciones y acciones. De igual forma, el investigador hace parte de la investigación y se afecta con ella lo que da lugar al despliegue de la experiencia.

Por otro lado, en términos de las posibilidades metodológicas propuestas desde la mirada arqueológica- genealógica se retoma como elementos claves para la sistematización y el trabajo documental, en primer lugar la consolidación del 
Bio - grafía. Escritos sobre la Biología y su Enseñanza. ISSN 2027-1034

Edición Extraordinaria. p.p. 657 - 664

Memorias del IX Encuentro Nacional de Experiencias en Enseñanza de la Biología y la

Educación Ambiental. IV Congreso Nacional de Investigación en Enseñanza de la Biología.

archivo, en este caso asumiendo el documento como práctica (Foucault, 2002), así se recurre a trabajos de grado, proyectos de facultad, guías de maestros entre otros. Estos documentos son tematizados en tres matrices, así el propósito es "desarticular los textos en sus temáticas centrales o principales para posibilitar de esa manera cruces horizontales entre documentos de diverso tipo y nivel de los cuales deberán salir agrupamientos temáticos en los que sea posible ubicar cada uno de los documentos trabajados." (Castro, 2007, p. 2). Posteriormente a la elaboración de matrices de realiza un ejercicio de lectura hipertextual y se identifican los elementos que son regulares y que a modo de enunciado pueden orientar la escritura.

Es importante mencionar que la elaboración de matrices se orienta de acuerdo al trabajo con tres conceptos metodológicos clave: saber, poder y subjetivación. Así las cosas, las relaciones entre éstos permite enunciar que el saber es aquello que circula y que genera prácticas, por su parte el poder no es pensado como algo que se posee y se transmite sino como relaciones de fuerza que se ejercen, se incitan o provocan. De este modo, lejos de pensar al poder como algo represivo, el poder es activo, es decir, produce sujetos. Es así, que el poder atraviesa todo el entramado social y se ejerce, no jerárquicamente, sino en redes formadas por un conjunto heterogéneo de elementos discursivos o no discursivos, el poder se constituye entonces en relación de fuerzas, "una situación estratégica en una sociedad en un momento determinado. Por lo tanto, el poder, al ser resultado de relaciones de poder, está en todas partes. El sujeto está atravesado por relaciones de poder, no puede ser considerado independientemente de ellas. El poder, para Foucault, no sólo reprime, sino que también produce: produce efectos de verdad, produce saber..." (Ibarra, 2008, p. 6).

En ese sentido, interesa aquí evidenciar cuáles son los saberes y prácticas que circulan sobre el Museo de Biología y las colecciones, las relaciones de poder que hacen que circulen estos y no otros saberes, que incitan éstos, que pueden llegar a crear y que efectos pueden tener en los procesos de constitución de sujetos, en este caso en particular, en la formación de maestros de biología.

\section{RESULTADOS Y DISCUSIONES}

Los hallazgos que se presentan a continuación se constituyen en un entramado inicial a modo de pistas para pensar la configuración del Museo Pedagógico de biología.

Tendencias encontradas alrededor de las prácticas del Museo de Biología y las colecciones

A partir de la revisión de los trabajos de grado, proyectos de facultad y otros 
Bio - grafía. Escritos sobre la Biología y su Enseñanza. ISSN 2027-1034

Edición Extraordinaria. p.p. 657 - 664

Memorias del IX Encuentro Nacional de Experiencias en Enseñanza de la Biología y la

Educación Ambiental. IV Congreso Nacional de Investigación en Enseñanza de la Biología.

documentos se pueden evidenciar tres grandes tendencias que dan cuenta de prácticas alrededor del Museo de Biología y de las colecciones del Departamento de Biología de la UPN. En esa medida, se encuentra:

1. Producción de materiales educativos como guías o catálogos ilustrados y bases de datos de tipo visual y de audio, que posicionan fuertemente el interés de los estudiantes por potencializar la enseñanza de la biología o un aspecto específico de ésta. Así mismo, se destaca la preocupación por fortalecer el Museo de Biología o Museo de Historia Natural del DBI, como se encuentra enunciado en algunos trabajos, en tanto se busca propiciar la sistematización y procesos de curaduría, organización y determinación de especies de organismos pertenecientes principalmente a colecciones como: entomológica, insectos acuáticos, botánica.

2. Los trabajos en relación a Sistemática y taxonomía se visibilizan con un número mayor de propuestas en comparación con las otras dos tendencias. Un aspecto recurrente de lo que allí se muestra es el interés por la identificación taxonómica de diferentes grupos de organismos, por otra parte, a partir de la identificación taxonómica surgen preocupaciones no solo de orden taxonómico sino de orden biogeográfico donde la distribución de estos grupos posibilita el conocimiento disciplinar en términos de lo ecológico y de su conservación. Sin embargo, estos estudios que se pueden considerar de "línea base" presentan constantemente la preocupación por pensar el impacto de dichos organismos en el escenario educativo del departamento de biología, toda vez que se trata de fortalecer, por ejemplo, el Cepario de Biotecnología, la colección de Insectos acuáticos, la colección entomológica y el herbario como posibilidades para impactar el desarrollo del Proyecto Curricular de Licenciatura en Biología.

3. Las Propuestas Pedagógicas evidencian la urgencia de destacar la importancia y el valor educativo del Museo de Biología e historia natural como espacios académicos para la formación de los licenciados en biología; además de aportar elementos justificativos que fomenten el uso educativo de las colecciones biológicas de la Universidad Pedagógica Nacional. Dichas propuestas son pensadas para deslocalizar el lugar común que tienen los museos y colecciones, y posicionarlos como lugares de comprensión de la vida y lo vivo. Así mismo, se piensa a través de estas propuestas que la dimensión cultural puede llegar a situar las colecciones como una posibilidad de reconocimiento de saberes ancestral donde la cartografía social tiene un papel importante para la configuración de estas. 
Bio - grafía. Escritos sobre la Biología y su Enseñanza. ISSN 2027-1034

Edición Extraordinaria. p.p. 657 - 664

Memorias del IX Encuentro Nacional de Experiencias en Enseñanza de la Biología y la

Educación Ambiental. IV Congreso Nacional de Investigación en Enseñanza de la Biología.

\section{Elementos para pensar la Configuración del Museo de Biología}

A partir de los primeros hallazgos y de las discusiones iniciales realizadas en el marco del Proyecto de Investigación, se encuentran en construcción varias apuestas a propósito de la comprensión de Museo Pedagógico de Biología. Así en primer lugar, vale la pena enunciar que el museo se configura más allá del lugar donde se guardan determinados objetos o colecciones, para este caso, museo se constituye en un espacio dinámico de pensamiento que incita a pensar cuales han sido los procesos de formación de maestros de biología, pues el objeto "expuesto" se asume como práctica relacionada con apuestas en enseñanza de la biología, y por tanto su puesta en escena tiene que ser de manera contextualizada, de tal forma, que permita evocar la experiencia que su historia le confiere. Así las cosas, retomando a Herrera \& Ojeda (2008) hablar de museo implica dar lugar para el despliegue de la memoria, pero no en términos del recuerdo alrededor del objeto, sino como memoria relacional, viva y fluctuante que posibilita, en este caso, historiar unas prácticas alrededor de la enseñanza de la biología fuera de una pretendida linealidad, que da lugar a las voces de maestros que han construido objetos y posibilidades a partir de ellos, o diseñado material de orden pedagógico o didáctico, develando con ello la experiencia que cruza su ser y quehacer.

En ese sentido, el museo así pensado desde la noción de memoria permite realizar una mirada a lo que somos y hemos sido como maestros de biología, pero no para legitimarlo sino para interrogar cuáles son las prácticas alrededor de la formación de maestros y como se entiende la enseñanza de la biología desde otros escenarios. Finalmente, lo pedagógico del museo de biología tiene que ver con la posibilidad de que los objetos se posicionen desde la memoria y la historia de las prácticas de enseñanza de la biología, para así complejizar la mirada alrededor de los procesos de formación de maestros.

\section{CONCLUSIONES}

- El historiar las prácticas de enseñanza alrededor de las colecciones y el museo en el Departamento de Biología, brinda elementos para ampliar su comprensión; pues así se observa que el museo puede situarse como espacio dinámico que permite generar procesos de pensamiento alrededor de la enseñanza de la biología y la formación de maestros.

- Las tendencias alrededor de las prácticas del museo y la colección se centran principalmente en la taxonomía y curaduría de organismos, la 
Bio - grafía. Escritos sobre la Biología y su Enseñanza. ISSN 2027-1034

Edición Extraordinaria. p.p. 657 - 664

Memorias del IX Encuentro Nacional de Experiencias en Enseñanza de la Biología y la Educación Ambiental. IV Congreso Nacional de Investigación en Enseñanza de la Biología.

generación de material educativo y las propuestas de orden pedagógico. Estas tendencias permiten posicionar las colecciones y el trabajo alrededor de ellas, como oportunidad para pensar estos espacios, no como lugares "muertos" o estáticos, sino por el contrario como escenarios para conservar la vida de los organismos.

- Los primeros hallazgos permiten tejer elementos para configurar el Museo Pedagógico de Biología, siendo importante destacar las construcciones alrededor de la memoria, la cual se instala no como un proceso estático sino dinámico y fluctuante permitiendo la movilización de pensamiento con el fin de deslocalizar las prácticas en relación con la enseñanza de la biología y problematizar su lugar en lo actual.

\section{BIBLIOGRAFÍA}

Castro, J. O. (2007). La ficha analítica y la ficha temática: Soportes para el trabajo documental. Documento de trabajo. Bogotá.

Foucault, M. (2002). La arqueología del Saber. Siglo XXI Editores. Argentina

Herrera, C; Ojeda, C. (2008). Memoria e Historia. Recuperar, Reflexionar y formar desde el Museo Pedagógico Nacional. Rev. Pedagogía y Saberes N.ㄹ 28. Facultad de Educación. Bogotá: Universidad Pedagógica Nacional. pp. 91-98.

Ibarra, J. (2008). Foucault y El Poder. Diatriba al Derecho, la Razón de Estado y los aparatos Disciplinarios. Valparaíso.

Proyecto Curricular Licenciatura en Biología. (2000). Departamento de Biología. Bogotá: Universidad Pedagógica Nacional. 